UNIVERSITY OF GOTHENBURG

SCHOOL OF BUSINESS, ECONOMICS AND LAW

WORKING PAPERS IN ECONOMICS

No 639

\title{
Social Distortion in Weight Perception: A Decomposition of the Obesity Epidemic
}

Paolo Nicola Barbieri

December 2015

ISSN 1403-2473 (print)

ISSN 1403-2465 (online) 


\title{
Social Distortion in Weight Perception: A Decomposition of the Obesity Epidemic
}

\author{
Paolo Nicola Barbieri*†
}

December 2, 2015

\begin{abstract}
This paper examines the influence of social norms on obesity. We develop a concept of social norm related to social distortion in weight perception developed through shared experiences in a common social environment with a high prevalence of obesity. The theoretical model show that when obesity is common it less likely to be recognized as a problem by mitigating individual's health concerns. We prove that our empirical measures of such a social component are significant in influencing individual weight using regional data from the Health Survey for England. We use the marked difference in obesity rates between 2002 and 2006 to undertake a Fairlie decomposition analysis. Our findings suggest that when we exclude social norms our estimates explain less than $50 \%$ of the obesity gap. When we include the social norms our estimates explain between $50 \%$ and $80 \%$ of the overall obesity gap. By stratifying the result by gender we are able to prove that men are more susceptible to social distortion, especially the low-skilled. Medium- and low-skilled women, similarly, are more susceptible to environmental pressure than highly-skilled women, who result as being completely unaffected by it. Men are also affected by a broader set of environmental pressures, with respect to women. Overall, these results suggest that an individual's concern over his or her body weight is closely related to the actual weight of his or her reference network.
\end{abstract}

JEL-Classification: D91, I10, I12, I18, Z13

Keywords: Obesity, non-linear decomposition, social norm

${ }^{*}$ I am thankful to Alessandro Saia, Francesca Barigozzi, Giulio Zanella, Daniele Fabbri, Chiara Monfardini, Franco Sassi, Marion Devaux, Davide Dragone, Giacomo Pasini, Giovanni Ponti, Matteo Lippi Bruni, and seminar participants at University of Bologna, OECD for insightful comments

${ }^{\dagger}$ Corresponding author. Centre for Health Economics, University of Gothenburg, Vasagatan 1, S-41124 Goteborg, Sweden E-mail address: paolonicola.barbieri@economics.gu.se 
"The social aspects of obesity may have a [social] multiplier effect on the growth of obesity. When obesity is relatively rare, it is considered abnormal and repulsive, and this negative response helps to keep it in check. As obesity begins to rise, the negative image of obesity becomes less intense because obesity is now more common."

Philipson and Posner, JEL (2008)

\section{Introduction}

Although obesity has, over the last two decades been a broad policy concern, the reasons for its longlasting incidence are yet to be entirely unraveled (Cawley, 2015, Popkin, 2007). Since the average weight gain has been too abrupt to be the result of a major genetic evolution several scholars have recently focused on other possible explanations such as: the decrease in real food prices (Goldman et al., 2011, Philipson and Posner, 2003, 2008), the reduction of the time cost of food (Cutler et al., 2003, Ruhm, 2012), technological changes due to economic growth (Huffman et al., 2010, Lakdawalla and Philipson, 2009) or the introduction of welfare-improving technological change (Lakdawalla et al., 2006). However, these explanations are able to explain just partially the so-called "obesity epidemic" (Cawley, 2015). Indeed, food prices and, most importantly, eating time and physical activity, declined substantially from the early 1970s though to the mid1980s due to technological innovations which allowed for cheap and energy-dense food, increases in restaurant supply $(\overline{R u h m}, 2012)$ and less physically demanding jobs (Philipson and Posner, 2003). These changes may have been the starting causes of an imbalance between calorie intake and calorie expenditure, although, given the fact that prices varied little afterwards, they cannot, alone, entirely explain the full growth of obesity (Christian and Rashad, 2009).

Recently, peer effects and social norms have been characterized as important pathways for the biological and behavioral spread of excessive body weight. Norms and customs developed through shared experiences in a common social environment can exert significant influence on behavior. When the majority of one's peers are obese and such condition is common, imitative obesity could represent an important contributor to weight-related norms. This view argues that weight gain exhibits a sort of 'social contagion' within friends and family networks (Christakis and Fowler, 2007, Trogdon and Allaire, 2014). This type of contagion may have effect because judgments regarding body size depend on the actual size of one's friends or on the prevalence of overweight/obese peers. Obesity is, in fact, spread through social ties and peers since having social contacts who are obese could change a person's tolerance of obesity or influence his or her adoption of specific behavior Ali et al., 2012, Christakis and Fowler, 2007, Halliday and Kwak, 2009, Trogdon et al. 2008). Furthermore, eating patterns and relative satiation also appear to be affected by an individual's surroundings, as consumption is adjusted according to the body type of others (McFerran et al., 2010a b).

For these reasons, social interaction has been explored as one of the possible channels of the obesity epidemic. Empirical literature has mostly focused on disentangling the relationship between individuals' perception of their own body weight with respect to their reference group, either because of social norms 
(Etilé, 2007, Gil and Mora, 2011) or because overweight perceptions and dieting are influenced by a person's relative BMI (Blanchflower et al., 2009, Oswald and Powdthavee, 2007). Theoretical literature has tried to provide some general predictions in order to define the form of such a social component and to provide a possible mechanism through which peers might be able to influence individual weight actions such as: exogenous social norms (Burke and Heiland, 2007, Dragone and Savorelli, 2012), intertemporal social pressure and appearance evaluations (Strulik, 2014, Wirl and Feichtinger, 2010) or more general interplay between economic, social and psychological factors (Reich and Weibull, 2012).

This paper highlights another channel: social distortion in weight perception, such that if being overweight is a widely spread phenomenon, it is less likely to be recognized as a problem by mitigating its health concerns. In the United States, for example, of the many adults who meet the conventional body mass index (BMI) standard for being overweight $(B M I \geq 25)$, few have perceived themselves thus (Burke et al., 2009, Paeratakul et al., 2002, Rand and Resnick, 2000), particularly between 1988 and 1994 and 1999 and 2004 when the increase in overweight people/obesity was more pronounced (Johnson-Taylor et al., 2008). These underestimations are usually described as misperceptions; however, this fails to take into account the social dimension and the fact that the secular increase in adult mean BMI is the main contributor to changes over time in weight perceptions. In fact, these underestimations correspond to the actual prevalence of overweight people in the representative group, and, rather than being misperceptions, these judgments are more likely to be accurate representations of the social context. Additionally, adults of all races, genders and weight "tend to see weight problems everywhere but in the mirror", so that they see the national weight problem as being greater than the individual weight problems of their friends and relatives (Taylor et al. 2006) Mistaken perceptions have been linked to dangerous behavior, whereas accurate perceptions have been associated with appropriate weight goals (Keightley et al. 2011). We assume that individuals are less likely to perceive themselves as being at risk because the whole categorization of weight has evolved due to a constant exposure to a growing population of overweight individuals so that current generations are more likely to misperceive their own weight (Ali et al., 2011, Burke et al., 2009, Maximova et al., 2008).

The present paper analyses a theoretical and empirical model with a social component by considering an interaction between one's own utility and the social prevalence of excessive weight in the society as a whole, which represents a social norm. Depending on the prevalence of obesity, the individual's perception of what is a healthy weight will be to some degree distorted, and the individual will therefore risk converging towards an unhealthy weight due to a social norm related to the actual realization of weight in the reference network. The theoretical model provides an explanation for the recent increase in obesity, relating it to the evolution of social approval of excessive weight which is affecting individuals' health concerns. As society is more indulgent towards obesity, it will be less commonly perceived as a health problem and society will converge to an overweight equilibrium. The empirical model using pooled data from the Heath Survey of England (HSE) from 2002 to 2006 tests this conclusion by establishing several social norms based on regional Health

\footnotetext{
${ }^{1}$ In a survey, made in 2006 in United States by the Pew Institute (Taylor et al., 2006) the percentage of individual reporting themselves as 'very overweight' is almost $25 \%$ lower than the actual trend and more than half of the respondent reported to have a weight 'just about right' when they should be categorized as 'overweight' so that they.
} 
Authorities. Using a Fairlie decomposition we are able to estimate that the $22 \%$ increase in obesity rates between $2002(20.2 \%)$ and 2006 (24.7\%), which accounts for a 5\% percentage point increase, was due to such social norms. Our findings suggest that when we exclude the social norms our estimates explain $40 \%$ of the obesity gap. When we include the social norms our estimates explain between $50 \%$ and $80 \%$ of the obesity gap. We also find that men have greater increases in obesity rates with respect to women, and that they are more susceptible to social distortion, especially low-skilled ones. Medium- and low-skilled women, similarly, are more susceptible to environmental pressure than highly-skilled ones, who are completely unaffected by it. While men are affected by all social norms used, women are found to be mainly affected by the one associated with their closest peers, in terms of demographic characteristics. Overall these results show the need to reduce misperception and enhance health education in order to provide social-invariant weight categorization to be followed to sustain and promote excessive weight reduction.

\section{Big and Beautiful: Has Overweight Become the "New Normal"?}

Norms and customs develop through shared experiences in a common social environment and can exert important influences on behavior. Individuals interact with each other and therefore influence other's behavior, for example through social norms. Regarding bodyweight, when the majority of peers are overweight and such condition is commonly observed, such social norm could distort health concerns regarding excessive body weight. Thus, many adults who meet the conventional BMI standard for overweight $(B M I \geq 25)$ might underestimate their weight category ${ }^{2}$, These underestimates of BMI categories are usually described as misperceptions; however this fail to recognize their social dimension. In fact rather than misperception these judgments are more likely accurate representations of the social context against the prevailing environmental distribution of weight.

Misperception of bodyweight is higher when an individual considers just his or his peers weight rather than the entire population. From a 2006 U.S. survey (Taylor et al., 2006) it emerged that Americans "tend to see weight problems everywhere but in the mirror" (p. 1). Most Americans do believe that getting fat is a serious policy concerns, however when they think about it they tend to use different scale for different people.

"Nine-in-ten American adults say most of their fellow Americans are overweight. But just sevenin-ten say this about "the people they know." And just under four-in-ten (39\%) say they themselves are overweight."

It seem that perception about weight are indeed shaped by the weight of the individual himself and his peers. Among adults of all races, genders and weights, however, one relationship is constant - people tend to see the national weight problem as being greater than the weight problems of their friends and relatives. In the survey, the percentage of individual reporting themselves as 'very overweight 3 is almost $25 \%$ lower than the

\footnotetext{
${ }^{2}$ Underestimates of BMI weight categories are more frequent in overweight men than women, in Blacks and Hispanics compared to Whites, and in those with less education (Bennett and Wolin (2006), Dorsey et al. (2009)).

${ }^{3}$ Obesity was omitted as a categorisation.
} 
actual trend. Moreover more than half of the respondent reported to have a weight 'just about right' when they should be categorised as 'overweight'

A 2008 investigation (Johnson-Taylor et al. 2008) on the evolution of weight perception in the United States from 1988-1994 (NHANES III) to 1999-2004 (NHANES) reported that the increases in overweight/obesity between these two survey years was also associated with fewer overweight individuals perceiving themselves as overweight. In addition to such evidence, Burke et al. (2009) examined the two most recent National Health and Nutrition Examination Survey (NHANES) of 1988-1994 and 1999-2004 testing the hypothesis that secular increases in adult mean BMI contributed to changes over time in weight perceptions. Overweight self-classification is significantly lower in the recent surveys for both women and men, controlling for objective weight status and other factors. Most importantly changes in body fat percentage and waist circumference do not account for the changes in weight classification between surveys.

Weight misperception could represents a very serious thread also for adolescence. Maximova et al. (2008) find that adolescents who live in environments in which the people they see on a daily basis (e.g. parents, schoolmates) are overweight/obese may develop inaccurate perceptions of what constitutes appropriate weight status. This misperception is stronger for overweight adolescence that for non-overweight. Given that adolescence overweight tracks into adulthood (Deshmukh-Taskar et al., 2005), if misperception is developed during childhood is very likely that it will be also carried on during adulthood.

In conclusion, there seems to be a substantial evidence of a generational shift in social norms related to body weight. As a result, people may be less likely to desire weight loss than previously, limiting the effectiveness of public health campaigns aimed at weight reduction. Subjective threshold for overweight increased during the last 20 years, consistent with theories of endogenous weight norms (Dragone and Savorelli, 2012, Etilé, 2007). Moreover individuals appears also to look at their immediate surrounding with a less strict judgment with respect to body weight highlighting the tendency to find comfort for their own "overweightness" in their social sorroundings.

\section{The Model}

\subsection{Utility function and the threshold effect}

Consider an individual $i$ being part of group $G$, with mass equal to 1, depending on his demographic, geographical or economic characteristics. The individual utility function of agent $i$ belonging to group $G$ depends on food consumption, $c_{i}(t) \geq 0$ and bodyweight, $w_{i}(t)>0$, according to the following quadratic specification (Dragone and Savorelli (2012))

$$
U_{i}\left(c_{i}, w_{i}\right)=c_{i}\left(c_{i}^{F}-\frac{c_{i}}{2}\right)-\beta \frac{\left(w_{i}-w_{i}^{H}\right)^{2}}{2}-\gamma \frac{\left(w_{i}-w^{G}\right)^{2}}{2}
$$

The first term represents direct utility from food consumption and the parameter $c_{i}^{F}$ is the individual's satiation point. This satiation level can be interpreted both from an economic point of view as the solution 
of a standard constrained optimisation problem where the agent much choose between two good 4 , food and non-food; or alternatively, satiation may also be literally interpreted, in which case restraining food intake below the satiation level can be interpreted as dieting. We say agent $i$ is underconsuming (or on a diet) if she eats less than her satiation level, and she is overconsuming if she eats more.

The second term represents the health consequences of being either overweight or underweight, occurring when a person's BMI is higher than 25 or lower than 18.5. When the individual BMI is between 18.5 and 25, a person is considered to have a normal weight which we will assume to be associated with $w_{i}^{H}>0$, maximising individual's health condition. The health consequence of bodyweight are summarised by the disutility cost the agent suffers if her body weight $w_{i}$ is different from her healthy weight. When $w_{i}<w_{i}^{H}$ we say that the agent is underweight, while, when $w_{i}>w_{i}^{H}$ we say that the agent is overweight.

The third term is related to the existence of a socially desirable body weight, determined by a social norm $w^{G} \geq 0$, which will be exogenously determined by the group $G$ and will represents a standard for all group's member:5. This weight will be assumed to be different, due to individual's heterogeneity with the individual healthy weight. Having a bodyweight different from such social reference ail be costly for the agent. We will assume that $w^{G}>w^{H}$ in order to rule out any cases of pressure to conform to underweight.

Moreover, we will assume that the parameters measuring the health and social cost from deviations from $w^{H}$ and $w^{G}$ (i.e. weight sensitivity) will not be exogenous parameters. Assume that the proportion of individual with weight equal or lower than $w^{G}$ is

$$
P\left(w \leq w^{G}\right)=F_{w}\left(w^{G}\right)=\int_{\underline{w}}^{w^{G}} f_{w}(t) d t
$$

where $\underline{w}$ represent the lower bar of weight distributions, then

$$
F\left(w^{G}\right)=1-F_{w}\left(w^{G}\right)
$$

represents the proportion of individual with weight higher than $w^{G}$. The costs from deviating from either $w^{H}$ or $w^{G}$ will be relates to the distribution of weight in the reference group, in the following way

$$
\beta=\left(1-F\left(w^{G}\right)\right) \quad \gamma=F\left(w^{G}\right)
$$

Equation (2) highlights the fact that, as the average weight increases, the agent becomes less concerns regarding the health consequence of excessive weight and more prone to adjust to the social norm, $w^{G}$.

Definition 3.1 (Threshold Effect). We define the threshold effect as the case in which overweight peers represents a majority in $G$, such that $\beta<\gamma$.

\footnotetext{
${ }^{4}$ Hence, the satiation point depends on the agent's preferences, on her income, and on market prices, i.e. $c^{F}=c^{F}\left(p_{c}, p_{x}, M\right)$, where $p_{c}$ and $p_{x}$ are the market prices and $M$ is income. An agent eating beyond (below) satiation can thus be interpreted as eating more (less) food than required by the optimality condition where the marginal rate of substitution equals the price ratio.

${ }^{5}$ We assume that the distribution of weight is a datum to the individual, so that if she acquire a large/small $w_{i}$ this has no effect on $w^{G}$.
} 
Such threshold effect is not creating a monotonic increases in bodyweight, rather a sensitivity adjustment over what represents the new norm (Blok et al., 2013).

\subsection{The determinants of bodyweight}

Regarding the intertemporal evolution of bodyweight, the agent knows that he cannot choose directly his bodyweight, but that it will be endogenously determined by the imbalance between calories intake and expenditure. Since the largest source of calories expenditure is due to the basic functioning of human body (i.e. organs, tissues), and it is measured by the basal metabolic rate, which is the energy expended at rest and is directly influenced on bodyweight along with individual's characterises we assume that body weight changes over time according to the following law of motion (Broeder et al., 1992, Mifflin et al., 1990):

$$
\dot{w}_{i}(t)=c_{i}(t)-\delta w_{i}(t)
$$

where $\delta \in(0.1)$ is a parameter reflecting the effect of weight on the burning of calories, or metabolism. Thus $\delta w_{i}(t)$ represents energy expenditure due to basal metabolic rate.

\section{Optimality conditions and stability}

The individual's goal is to maximize his intertemporal utility by choosing the amount of food consumption, which in turn will affect his bodyweight. Given an infinite time horizon $\sqrt{6}$ and a positive discount rate, $\rho$, the individual's problem can be written as follows:

$$
\begin{gathered}
\max _{c(t)} \int_{0}^{\infty} e^{-\rho t}\left[c_{i}(t)\left(c_{i}(t)^{F}-\frac{c_{i}(t)}{2}\right)-\left(1-F\left(w^{G}\right)\right) \frac{\left(w_{i}(t)-w_{i}^{H}\right)^{2}}{2}-F\left(w^{G}\right) \frac{\left(w_{i}(t)-w^{G}\right)^{2}}{2}\right] d t \\
\text { subject to } \dot{w}(t)=c(t)-\delta w(t) \\
w(t), c(t) \geq 0 \\
w(0)=w_{0}, \text { given }
\end{gathered}
$$

Proposition 4.1. The intertemporal problem (4)-(7) is associated with:

1. A unique steady state level of bodyweight and food consumption

$$
\begin{aligned}
& c^{s s}=\delta \frac{\delta w^{F}(\delta+\rho)+F\left(w^{G}\right) w^{G}+\left(1-F\left(w^{G}\right)\right) w^{H}}{A} \\
& w^{s s}=\frac{\delta w^{F}(\delta+\rho)+F\left(w^{G}\right) w^{G}+\left(1-F\left(w^{G}\right)\right) w^{H}}{A}
\end{aligned}
$$

\footnotetext{
${ }^{6}$ We refer to Dragone et al. (2015), Yaari (1965) for the result relating our infinite horizon case with a similar problem with an uncertain terminal date $T$.
} 
where $A=\delta(\delta+\rho)+1$.

2. The steady state has a saddle point stability.

3. In the steady state the agent is overweight if $w^{G}>\tilde{w}$ and overconsuming if $w^{G}>\tilde{\tilde{w}}$

Proof. See Appendix A

Looking at equation $(9)$ we can notice that

Corollary 4.1. Whenever overweight peers represents a majority in the reference group, the individual will converge to a higher bodyweight.

Proposition 4.2 (Social Distortion in Weight Perception). An increases in the proportion of overweight peers lead to an increases in bodyweight

Proof. If we take the derivative of Equation (9) with respect to $F\left(w^{G}\right)$ we have

$$
\frac{\partial w^{s s}}{\partial F\left(w^{G}\right)}=\frac{w^{G}-w^{H}}{A}
$$

Given that $A>0$ Equation 10 is always greater than zero, provided that $w^{G}>w^{H}$, i.e. when my peers are overweight.

Proposition 4.2 describes a situation in which due to the social interaction with overweight peers, the individual grows accustom to observe such prevalence and thus he perceived it as a social norm to conform to. This will affect his sensitivity over which weight is suppose to represents the most derivable standard to conform to, and, as the majority of his/her reference group gets heavier the more he will converge to $w^{G}$.

\section{$5 \quad$ Data and Variables}

\subsection{Data Source}

We use pooled data from five rounds (from 2002 to 2006) of the Health Survey for England (HSE), which is a cross-sectional survey followed by a nurse's visit. The HSE is a nationally representative survey of individuals aged two years and over living in England. Every year a new sample is drawn and respondents are interviewed on a range of topics including demographic and socio-economic indicators, general health and psycho-social indicators. Additionally, there is a follow up visit by a nurse where various physiological measurements are taken including height and weight.

The proportion of the sample in each body weight category is in Table 1 . Only $38 \%$ of the sample are in the healthy weight category while $60 \%$ are either obese $(23 \%)$ or overweight $(37 \%)$. Overweight and obese individuals are older, reporting lower general health but with similar marital status. Obese individuals seem to live in authorities characterized by a higher proportion of obese peers.

[Table 1 about here.] 


\subsection{Obesity and Covariates}

\subsubsection{Dependent Variable: Obesity Measure}

The obesity measure is computed for each respondent from the height and weight values obtained during the nurse's visit. One useful feature of this dataset is that height and weight are measured by a nurse rather than being self-reported, thus reducing the likelihood of errors. Obesity is measured as a dummy variable taking a value of one if the individual has a BMI over 30 and zero otherwise.

\subsubsection{Covariates}

We use a set of covariates grouped in six categories.

Socio-Economic Status: Socio-economic status is measured using the skill level of the individual defined either as (1) low (i.e. semi-skilled and unskilled manual); (2) medium (i.e. skilled manual and non-manual) and (3) high (i.e. professional and managerial). In order to focus on individuals of a working age and to avoid any bias due to retirement weight distortion, we restricted our sample to individuals between 18 and 65 years old.

Education Attainment: Educational attainment is a continuous variable measuring the age at which the respondent finished his or her full-time education at school or college minus four years; we also included its square.

Health Variables: Measures of self-reported general health, acute ill-health, longstanding illness and psycho-social health. Self-reported general health is a measure of subjective general health measured in five categories ranging from very good to very bad. Acute ill-health is measured by the number of days in the preceding two weeks that the respondent had to cut down on the things they usually do because of illness or injury. In terms of longstanding illnesses respondents are asked whether they have an illness, a disability or an infirmity that has troubled them over a period of time, and its type by broad disease code. Limiting longstanding illness is categorized by whether any of these illnesses limit respondents' activities in any way. Psycho-social health is measured by a GHQ-12 score, where higher values indicate more severe psycho-social problems.

Housing, marriage and family size: The HSE collects information on respondents' marital status and housing tenure. We also controlled for the number of infants living in the household aged between zero and one year and the number of children aged between two and 15 years living in the household.

Food Prices: Monthly food and non-alcoholic beverages consumer price index (CPI) that measures changes in the price of food over time, derived from the Food and Agriculture Organization of the United Nation Statistics (FAOSTAT).

Additional control: Such as: gender; age; ethnicity; eating habits (number fruit and vegetable servings per week). 


\subsection{Social Distortion Proxies}

Health Authorities were once part of the structure of the National Health Service (NHS) in England, each one was responsible for enacting the directives and implementing fiscal policy as dictated by the Department of Health at a regional level. They were reformed in 2001 7 and then abolished in 2006 8 . During the period 2002-2006 they remained unchanged in number and size so we decide to focus on this time period in order to exploit their physical dimension and examine how the average social distortion in health in Health Authorities affected the spread of obesity. We therefore constructed the following three (continuous) variables in order to proxy social distortion:

- Average BMI in the Health Authorities where the respondent lives: Computed as the average BMI of the Health Authority where the individual lives;

- Demographic Group: Health Authority average BMI of a reference group, defined by gender and age category divided into three thresholds (18-30, 31-41 and 42-65);

- Percentage of obese peers in the Health Authorities where the respondent lives: Computed as the average percentage of obesity in the Health Authority where the individual lives.

We assumed that social distortion in health is spread by social norm and tried to estimate it using the three variables above. Conditional to the other covariates, these factors are significant for two reasons. First of all they represent three measures of environmental influences that might affect body weight, by summarizing the food intake and physical habits of the local population which affect the ego's decision. Second they also affect one's own obesity via what Manski (1993) identify as "endogenous effect". This is the effect that peer obesity has on individual obesity all else being equal. Keeping all other elements constant peer obesity has an effect on individual obesity because it reflects a social norm to conform to.

Figure 1 shows the area-level incidence of obesity in 2002 (top panel) and in 2006 (bottom panel), from which we can see that between 2002 and 2006 obesity rates grew quite substantially in almost every one of the 28 Health Authorities examined, accounting for a 4.5\% point percentage increase in obesity between 2002 $(20.2 \%)$ and $2006(24.7 \%)$, resulting in an overall increase in obesity rates over this period of $22 \%$.

[Figure 1 about here.]

\section{Obesity increases over time: Within-Group or Across-Group Changes?}

\subsection{Fairlie's Decomposition}

As pointed out earlier, over the period between 2002 and 2006 obesity rates increased by almost five percentage points, accounting for an overall increase in obesity rates in this period of $22 \% 9$. Given that

\footnotetext{
${ }^{7}$ When the existing regional health authorities were renamed and merged to form the 28 new strategic health authorities.

${ }^{8}$ After 2006 the NHS decided to reorganized the Health Authorities and to reduce their number to ten.

${ }^{9}$ This figure are in line with the OECD estimates (Sassi and Devaux, 2012)
} 
this increase is quite striking for such a short time period we argue that this increase may be due to the increased weight of the population, as overweight becomes a new standard and a social distortion in weight is created, rather than to an underlying demographic feature of the English population arising during this period which could have caused large changes for certain demographic groups, while other groups' body composition remained unchanged.

In order to quantify the contribution that social norm had on obesity rates over time we employ Fairlie (1999, 2005) decomposition technique as it is more suitable for calculating gaps for binary variables (Font et al. 2010). Following Fairlie 1999,2005$)$ the decomposition for a non-linear equation of the type $p(y=1)=F(x \hat{\beta})$ can be expressed as

$$
\bar{y}^{E}-\bar{y}^{L}=\left[\sum_{i=1}^{N^{E}} \frac{F\left(x^{E} \hat{\beta}^{E}\right)}{N^{E}}-\sum_{i=1}^{N^{L}} \frac{F\left(x^{L} \hat{\beta}^{E}\right)}{N^{L}}\right]+\left[\sum_{i=1}^{N^{L}} \frac{F\left(x^{L} \hat{\beta}^{E}\right)}{N^{L}}-\sum_{i=1}^{N^{L}} \frac{F\left(x^{L} \hat{\beta}^{L}\right)}{N^{L}}\right]
$$

where $\bar{y}^{i}$ is the average obesity probability in period $j(j=E, L$ for early and late period, respectively), $x_{j}$ is the set of average values of the independent variables used is period $j, \hat{\beta}^{j}$ is the coefficient estimated for period $j, F$ is the cumulative distribution function from a standard normal or a logistic distribution and $N$ refers to the sample size in each period. The first term in brackets in equation (11) shows the part of cross-time difference that is due to group differences in the distribution of characteristics of the $x_{j}$, referred as the "explained part", while the second term in brackets represents the portion of the cross-time differences due to differences in coefficients to the exogenous covariates but it also captures differences in unobservable endowment, this is why is referred as the "unexplained part". Similarly the non-linear decomposition can be written as

$$
\bar{y}^{E}-\bar{y}^{L}=\left[\sum_{i=1}^{N^{E}} \frac{F\left(x^{E} \hat{\beta}^{L}\right)}{N^{E}}-\sum_{i=1}^{N^{L}} \frac{F\left(x^{L} \hat{\beta}^{L}\right)}{N^{L}}\right]+\left[\sum_{i=1}^{N^{E}} \frac{F\left(x^{E} \hat{\beta}^{E}\right)}{N^{E}}-\sum_{i=1}^{N^{E}} \frac{F\left(x^{E} \hat{\beta}^{L}\right)}{N^{E}}\right]
$$

Where in this case the estimated coefficients for the late period $\hat{\beta}^{L}$, are used as weights to calculate the first term of the decomposition, and the early distribution of average characteristics is used as weights for the second term. Given that the two decomposition in Equations 111 and 12 provide different estimates, to avoid this problem we used the coefficient estimates from a pooled sample over all cases to weight the unexplained part of the decomposition (Oaxaca and Ransom, 1994). According to Fairlie (2005), Equations (11) and (12) provide an estimation of the contribution that the explained and unexplained part have on the total difference between the two periods. To test our hypothesis we will concentrate on the contributions that the individual independent variables have on the probability of being obese with a particular interest in the social norm ones.

The calculation of the separate contributions of the individual independent variables (or group of covariates) is not direct. One has to assume that $N^{E}=N^{L}$ and $\hat{\beta}^{*}$ is the probit coefficient estimates from a pooled sample, then the individual contribution of regressor $x_{k}$ to the cross-time obesity difference can be written 
as,

$$
\frac{1}{N^{L}} \sum_{i=1}^{N^{L}} F\left(\hat{\alpha}^{*}+x_{k i}^{E} \hat{\beta}_{k}^{*}+\sum_{m \neq k} x_{m i}^{E} \hat{\beta}_{m}^{*}\right)-F\left(\hat{\alpha}^{*}+x_{k i}^{L} \hat{\beta}_{k}^{*}+\sum_{m \neq k} x_{m i}^{E} \hat{\beta}_{m}^{*}\right)
$$

Which means that the contribution of a particular variable to the difference is calculated by holding constant the contribution of the other variables ${ }^{10}$. Notice that the computation of equation (13) involves a one-toone matching of cases between the two groups $\left(N_{E}>N_{L}\right)$ and as they typically differ in size, then a large number of random sub-samples from the larger group are drawn. Each of these random sub-samples of the early sample is then matched to the late sample and finally separate decomposition estimates are calculated. The mean value of estimates from the separate decompositions is calculated and employed to derive the results for the entire early sample.

In order to control for the social factors related to Health Authorities which may be behind the increase in obesity rate in the period we included the full set of covariates. Firstly, we checked for the average obesity rate of the Health Authority in order to discover the influence of common regional effects affecting both genders. Secondly, we inserted a measure of social norm. We interpreted social norm as a behavioral regularity that can be measured by the behavior of a reference group, so that any deviation from the norm results in a cost. The downside is that we cannot distinguish contextual from endogenous effects (Manski, 1993).

Table 2 displays Fairlie's decomposition of the obesity gaps between 2002 and 2006, both excluding and including the controls for social social norm. Looking at the total explained difference column at the bottom of the table, we see that the results indicate that our model covariates explain less than $50 \%$ of the overall gaps (-0.0202) when no control for social norm is included. Conversely, these determinants explain between $50 \%$ and $80 \%$ of gaps when social norm is controlled for $(-0.0214 ;-0.341)$. When no social environment is included, time differences in demographic (e.g. age, gender, ethnicity) and education explain the majority of the gaps. However, when social norm effects are included in the model, they are able to explain up to the $30-40 \%$ of the obesity gap alone (See column (2) and (4) of Table 2 ) and, when we use the social norm defined by demographic groups it overrides the effects of all other variables. This shows that social norms play a non-negligible role in the recent spread of obesity in our sample

One possible interpretation for the 50\%-20\% change still left to be explained might be related to welfareenhancing technological improvements which may have reduced the health impacts of chronic conditions related to obesity (Lakdawalla et al., 2006), or to the increases of less physically demanding job (Philipson and Posner, 2003). Although we are considering a relative short time period and these changes took place several years ago, it is unlikely that things changed a great deal between the beginning and the end of our sample, but they might still have a higher effect on both periods due to some form of persistency effect

[Table 2 about here.]

\footnotetext{
${ }^{10}$ See Fairlie (2005) for a further discussion on how to compute standard errors
} 


\section{Stratification Analysis}

\subsection{Gender}

The results in Table 2 point to a significant contribution of social norms through the channel of social distortion in weight perception to the increase in obesity rates between 2002 and 2006 for the overall population. However, since there is well-established literature reporting a marked difference between the effect that peer pressure has among men and women 11 , we decided to stratify our results for gender. In order to check whether such an effect is different for each gender we estimated the same decomposition of Table 2 but split the sample into men and women. The results are shown in Table 3.

[Table 3 about here.]

The results show that obesity rates between 2002 and 2006 increased by $6 \%$ for men and by $3.2 \%$ for women, thus leading to a more pronounced increase in obesity rates for males than for females. Like Table 2. Table 3 also shows that after including social norm variables, the gap explained by our model increases from $35 \%$ (not displayed in Table 3) to 50-90\% for men and from 40\% (not displayed in Table 3) to 40-50\% for women. These results show that men are more subject to social distortion and environmental factors with respect to women. Although the total explained difference increased for both men and women after adding the environmental covariates we can see that while for men those variables are always statistically significant (Columns (1), (3) and (5) Table 3), for women only the demographic group social norm variable is significant (Columns (2), (4) and (6) Table 3), underlining the fact that women are influenced by a specific social norm channel directly related to their closest reference group, in terms of age.

\subsection{SES}

In addition to gender we investigated whether there was a possible difference in subjectivity to social norms with respect to socio-economic status. As for gender differences in obesity and health behavior we did this because there is well-established literature connecting health behavior to socio-economic status differences (e.g. income, job status, education) (Cawley, 2004, Cutler and Lleras-Muney, 2010, Finkelstein et al., 2005, Han et al. 2009, Rosin, 2008). Therefore we decided to divide our sample by socio-economic status according to the type of labor that the individual was doing at the time of the interview, rather than using income or education. To do so we stratified our sample based on occupational category by creating two main clusters:

1. High: Including professional and managerial workers;

2. Medium-Low: Including skilled, semi-skilled and unskilled manual workers.

\footnotetext{
${ }^{11}$ See Christakis and Fowler (2007), Etilé (2007), Font et al. (2010), Halliday and Kwak (2009), Mora and Gil (2012), Trogdon and Allaire (2014), Trogdon et al. (2008) among other.
} 
We decided to divide our sample in this way in order to isolate workers who work in non-manual versus manual labor and we also decided to pool medium- and low-skilled individuals due to a scarcity of data.

Tables 4 and 5 show the results for men using our three main social norm definitions. In both tables the obesity rate increase is higher for medium- and low-skilled men $(7 \%)$ than for skilled workers $(4 \%)$. The percentage of this increase explained by our covariates is marginally higher for highly-skilled workers (45-87-90\%) rather than medium- or low-skilled ones (45-70-80\%). After a closer look at the coefficients we notice that the effect of the social norm covariate, related to the demographic group, between the two SES is higher and more significant for medium- and low-skilled individuals (-0.0245) than it is for highly-skilled workers (-0.0142) and that all the other social norm coefficients are more significant for the medium- and low-skilled (See columns (1), (2), (3) and (4) of Table 4 and columns (1) and (2) 5). This indicates that our decomposition analysis might be more efficient in capturing the overall gap between obesity rates for highly-skilled individuals between 2002 and 2006, but medium- and low-skilled individuals were more subject to social norm and therefore more affected by social distortion in weight perception.

[Table 4 about here.]

[Table 5 about here.]

Tables 6 and 7 show the results for women. As with men obesity rates were higher for medium- and low-skilled workers $(4 \%)$ with respect to highly-skilled workers $(2 \%)$. As before our decomposition seems able to explain this gap better for medium- and low-skilled women (33-45-70\%) than for high-skilled ones (20-25-30\%). These results indicate that increasing obesity rates among highly-skilled women between 2002 and 2006 are not due to environmental factors but to some other socio-economic explanations unrelated to social norm. As for the results in 3 the only social environmental variable that was significant for mediumand low-skilled women is the one related to the demographic group (Column (2) Table 6).

[Table 6 about here.]

[Table 7 about here.]

\section{Conclusion}

The world is getting heavier and at frightening pace. More and more research is being devoted to disentangling the main components that contribute to the spread of obesity over time. The fall in effective and real food prices and a decrease in physical activity at work are two of the prominent explanations provided in current literature for such an abrupt change in the human phenotype. However, obesity continued to spread long after these changes in eating patterns and food prices occurred. A possible explanation for such longlasting effects might be related to certain social dynamics between individuals? reference groups.

This paper has explored the consequences on the equilibrium decision of a rational individual of the social pressure to conform (or not) to a given social norm arising in the society, which will modify his or 
her judgment of what represents a healthy weight. Depending on the prevalence of obesity the individual will have a distorted perception of which weight represents a healthy reference and which weight does not, thus risking converging to an unhealthy weight due to social pressure related to the actual realization of weight in the reference network, establishing a social norm. The theoretical model gives an explanation for the recent increase in obesity relating it to the evolution of social approval of excessive weight, which is affecting individual social image. As society is more indulgent towards overweight/obesity, the greater the misperception of it as a health problem and the more society will converge to an overweight equilibrium. The empirical model using pooled data from the Heath Survey of England (HSE) between 2002 and 2009 tested this conclusion by establishing a social norm based on regional Health Authorities affecting health sensitivity. Using a Fairlie decomposition, we are able to estimate that the $22 \%$ increase in obesity rates between 2002 $(20.2 \%)$ and $2006(24.7 \%)$, which accounts for a $5 \%$ percentage point increase in our sample, was due to social norm components. Our findings suggest that when we exclude social norms our estimates account for less than $40 \%$ of the obesity gap. When we include the social norms our estimates explain between $50 \%$ to $80 \%$ of the obesity gap. We also find that men experience greater obesity rate increases with respect to women and that they are more susceptible to social distortion, especially the low-skilled. Medium- and low-skilled women, similarly, are more susceptible to environmental pressure than highly-skilled ones, who are completely unaffected by them. While men are affected by a broader set of environmental pressures, women are found to be mainly affected by their close peers, in terms of demographic group. The policy implications will be to reduce misperceptions of obesity and enhance health education in order to provide social-invariant weight categorization to be followed along with a greater concern for excessive weight reduction as a serious health problem.

\section{A Proof of Proposition 4.1}

The associated current value Hamiltonian (dropping the time indexes for convenience) is

$$
H(c, w, \lambda)=c_{i}\left(c_{i}^{F}-\frac{c_{i}}{2}\right)-\left(1-F\left(w^{G}\right)\right) \frac{\left(w_{i}-w_{i}^{H}\right)^{2}}{2}-F\left(w^{G}\right) \frac{\left(w_{i}-w^{G}\right)^{2}}{2}+\lambda(c-\delta w)
$$

Given joint concavity the necessary and sufficient conditions for optimality are ${ }^{12}$

$$
\begin{aligned}
& H_{c}=0 \quad c^{F}-c+\lambda=0 \\
& \dot{\lambda}=\lambda\left(\rho+g_{w}\right)-H_{w} \\
& \dot{w}=c-\delta w
\end{aligned}
$$

\footnotetext{
${ }^{12}$ If the limiting transervality condition, $\lim _{t \rightarrow \infty} e^{-\rho t} \lambda(t) w(t)=0$, holds.
} 
Thus the system of differential equations characterising the equilibrium is the following

$$
\begin{aligned}
\dot{c} & =\left(c-c^{F}\right)(\rho+\delta)+\left(1-F\left(w^{G}\right)\right)\left(w-w^{H}\right)+F\left(w^{G}\right)\left(w-w^{G}\right) \\
\dot{w} & =c-\delta w
\end{aligned}
$$

For an internal steady state the following conditions must be satisfied

$$
\begin{array}{cc}
\dot{c}=0 & \left(c-c^{F}\right)(\rho+\delta)+\left(1-F\left(w^{G}\right)\right)\left(w-w^{H}\right)+F\left(w^{G}\right)\left(w-w^{G}\right)=0 \\
\dot{w}=0 & c=\delta w
\end{array}
$$

The Jacobian matrix associated with (10) is

$$
J=\left(\begin{array}{cc}
\delta+\rho & 1 \\
1 & -\delta
\end{array}\right)
$$

the saddle point stability is ensured by the fact that the trace is always positive while the determinant is always negative.

To verify the condition on being overweight observe that the difference between $w^{s s}-w^{H}$

$$
w^{s s}>w^{H} \Leftrightarrow \delta w^{F}(\delta+\rho)+F\left(w^{G}\right) w^{G}+\left(1-F\left(w^{G}\right)\right) w^{H}-w^{H}(\delta(\delta+\rho)+1)>0
$$

can be written as

$$
w^{G}>\frac{w^{H}\left[F\left(w^{G}\right)+\delta(\delta+\rho)\right]-w^{F} \delta(\rho+\delta)}{F\left(w^{G}\right)}=\tilde{w}
$$

Similarly if we take the difference $c^{s s}-c^{F}$ we verify the conditions for overconsumption

$$
c^{s s}>c^{F} \Leftrightarrow \delta\left(\delta w^{F}(\delta+\rho)+F\left(w^{G}\right) w^{G}+\left(1-F\left(w^{G}\right)\right) w^{H}\right)-\delta w^{F}(\delta(\delta+\rho)+1)>0
$$

can be written as

$$
w^{G}>\frac{w^{F}-\left(1-F\left(w^{G}\right)\right) w^{H}}{F\left(w^{G}\right)}=\tilde{\tilde{w}}
$$

\section{References}

Ali, M. M., A. Amialchuk, S. Gao, and F. Heiland (2012). Adolescent weight gain and social networks: is there a contagion effect? Applied Economics 44 (23), 2969-2983.

Ali, M. M., A. Amialchuk, and F. Renna (2011). Social network and weight misperception among adolescents. Southern Economic Journal 77(4), 827-842.

Bennett, G. G. and K. Y. Wolin (2006). Satisfied or unaware? racial differences in perceived weight status. International Journal of Behavioral Nutrition and Physical Activity 3(1), 40. 
Blanchflower, D. G., B. Landeghem, and A. J. Oswald (2009). Imitative obesity and relative utility. Journal of the European Economic Association 7(2-3), 528-538.

Blok, D. J., S. J. de Vlas, P. Van Empelen, J. H. Richardus, and F. J. van Lenthe (2013). Changes in smoking, sports participation and overweight: Does neighborhood prevalence matter? Health 8 place.

Broeder, C. E., K. A. Burrhus, L. S. Svanevik, and J. H. Wilmore (1992). The effects of either high-intensity resistance or endurance training on resting metabolic rate. The American journal of clinical nutrition 55(4), 802-810.

Burke, M., F. Heiland, and C. Nadler (2009). From "overweight" to "about right": evidence of a generational shift in body weight norms. Obesity 18(6), 1226-1234.

Burke, M. A. and F. Heiland (2007). Social dynamics of obesity. Economic Inquiry 45(3), 571-591.

Cawley, J. (2004). The impact of obesity on wages. Journal of Human Resources 39(2), 451-474.

Cawley, J. (2015). An economy of scales: A selective review of obesity's economic causes, consequences, and solutions. Journal of health economics 43, 244-268.

Cawley, J. and C. J. Ruhm (2011). The economics of risky health behaviors1. Handbook of health economics 2, 95.

Christakis, N. A. and J. H. Fowler (2007). The spread of obesity in a large social network over 32 years. New England journal of medicine 357(4), 370-379.

Christian, T. and I. Rashad (2009). Trends in us food prices, 1950-2007. Economics Éamp; Human Biology 7(1), 113-120.

Cutler, D., E. Glaeser, and J. Shapiro (2003). Why have americans become more obese? Technical report, National Bureau of Economic Research.

Cutler, D. M. and A. Lleras-Muney (2010). Understanding differences in health behaviors by education. Journal of health economics 29(1), 1-28.

Deshmukh-Taskar, P., T. Nicklas, M. Morales, S. Yang, I. Zakeri, and G. Berenson (2005). Tracking of overweight status from childhood to young adulthood: the bogalusa heart study. European Journal of Clinical Nutrition 60(1), 48-57.

Dorsey, R. R., M. S. Eberhardt, and C. L. Ogden (2009). Racial/ethnic differences in weight perception. Obesity 17(4), 790-795.

Dragone, D., F. Manaresi, and L. Savorelli (2015). Obesity and smoking: can we kill two birds with one tax? Health economics. 
Dragone, D. and L. Savorelli (2012). Thinness and obesity: A model of food consumption, health concerns, and social pressure. Journal of Health Economics 31(1), 243-256.

Etilé, F. (2007). Social norms, ideal body weight and food attitudes. Health Economics 16(9), 945-966.

Fairlie, R. W. (1999). The absence of the african-american owned business: An analysis of the dynamics of self-employment. Journal of Labor Economics 17(1), 80-108.

Fairlie, R. W. (2005). An extension of the blinder-oaxaca decomposition technique to logit and probit models. Journal of economic and social measurement 30(4), 305-316.

Finkelstein, E., C. Ruhm, and K. Kosa (2005). Economic causes and consequences of obesity. Annu. Rev. Public Health 26, 239-257.

Font, J. C., D. Fabbri, and J. Gil (2010). Decomposing cross-country differences in levels of obesity and overweight: does the social environment matter? Social Science \& Medicine 70(8), 1185-1193.

Gil, J. and T. Mora (2011). The determinants of misreporting weight and height: the role of social norms. Economics \& Human Biology 9(1), 78-91.

Goldman, D., D. Lakdawalla, and Y. Zheng (2011). Food prices and the dynamics of body weight. University of Chicago Press.

Halliday, T. J. and S. Kwak (2009). Weight gain in adolescents and their peers. Economics $\&$ Human Biology 7(2), 181-190.

Han, E., E. C. Norton, and S. C. Stearns (2009). Weight and wages: fat versus lean paychecks. Health economics 18(5), 535-548.

Huffman, W. E., S. Huffman, K. Rickertsen, and A. Tegene (2010). Over-nutrition and changing health status in high income countries. Frontier in Health Policy Research 13(1), 2.

Johnson-Taylor, W., R. Fisher, V. Hubbard, P. Starke-Reed, and P. Eggers (2008). The change in weight perception of weight status among the overweight: comparison of nhanes iii (1988-1994) and 1999-2004 nhanes. International Journal of Behavioral Nutrition and Physical Activity 5(1), 9.

Keightley, J., A. Chur-Hansen, R. Princi, and G. A. Wittert (2011). Perceptions of obesity in self and others. Obesity Research Eamp; Clinical Practice 5(4), e341-e349.

Lakdawalla, D. and T. Philipson (2009). The growth of obesity and technological change. Economics 83 Human Biology 7(3), 283-293.

Lakdawalla, D. N., T. J. Philipson, and J. Bhattacharya (2006). Welfare-enhancing technological change and the growth of obesity. In American Economic Review: AEA Papers and Proceedings, Volume 95, pp. $253-257$. 
Manski, C. F. (1993). Identification of endogenous social effects: The reflection problem. The review of economic studies 60(3), 531-542.

Maximova, K., J. McGrath, T. Barnett, J. O'Loughlin, G. Paradis, and M. Lambert (2008). Do you see what i see? weight status misperception and exposure to obesity among children and adolescents. International Journal of Obesity 32(6), 1008-1015.

McFerran, B., D. Dahl, G. Fitzsimons, and A. Morales (2010a). Ill have what shes having: Effects of social influence and body type on the food choices of others. Journal of Consumer Research 36(6), 915-929.

McFerran, B., D. Dahl, G. Fitzsimons, and A. Morales (2010b). Might an overweight waitress make you eat more? how the body type of others is sufficient to alter our food consumption. Journal of Consumer Psychology 20(2), 146.

Mifflin, M. D., S. St Jeor, L. A. Hill, B. J. Scott, S. A. Daugherty, and Y. Koh (1990). A new predictive equation for resting energy expenditure in healthy individuals. The American journal of clinical nutrition 51(2), $241-247$.

Mora, T. and J. Gil (2012). Peer effects in adolescent bmi: Evidence from spain. Health Economics.

Oaxaca, R. L. and M. R. Ransom (1994). On discrimination and the decomposition of wage differentials. Journal of econometrics 61(1), 5-21.

Oswald, A. J. and N. Powdthavee (2007). Obesity, unhappiness, and the challenge of affluence: Theory and evidence.

Paeratakul, S., M. A. White, D. A. Williamson, D. H. Ryan, and G. A. Bray (2002). Sex, race/ethnicity, socioeconomic status, and bmi in relation to self-perception of overweight. Obesity research 10(5), 345-350.

Philipson, T. J. and R. A. Posner (2003). The long-run growth in obesity as a function of technological change. Perspectives in biology and medicine 46(3), S87-S107.

Philipson, T. J. and R. A. Posner (2008). Is the obesity epidemic a public health problem? a decade of research on the economics of obesity. Journal of Economic Literature 46(4), 974-82.

Popkin, B. M. (2007). The world is fat. Scientific American 297(3), 88-95.

Rand, C. S. and J. L. Resnick (2000). The good enough body size as judged by people of varying age and weight. Obesity Research 8(4), 309-316.

Reich, B. and J. W. Weibull (2012). Obesity as a social equilibrium phenomenon.

Rosin, O. (2008). The economic causes of obesity: a survey. Journal of Economic Surveys 22(4), 617-647.

Ruhm, C. J. (2012). Understanding overeating and obesity. Journal of Health Economics. 
Sassi, F. and M. Devaux (2012). Obesity update 2012. OECD 2012; 1-7.

Strulik, H. (2014). A mass phenomenon: The social evolution of obesity. Journal of Health Economics 33(0), $113-125$.

Taylor, P., C. Funk, and P. Craighill (2006). Americans see weight problems everywhere but in the mirror. Philadelphia: Pew Foundation Social Trends Report.

Trogdon, J. G. and B. T. Allaire (2014). The effect of friend selection on social influences in obesity. Economics \& Human Biology.

Trogdon, J. G., J. Nonnemaker, and J. Pais (2008). Peer effects in adolescent overweight. Journal of health economics 27(5), 1388-1399.

Wirl, F. and G. Feichtinger (2010). Modelling social dynamics (of obesity) and thresholds. Games 1(4), 395-414.

Yaari, M. E. (1965). Uncertain lifetime, life insurance, and the theory of the consumer. The Review of Economic Studies, 137-150. 
Figure 1: Area Incidence of Obesity in England 2002-2006
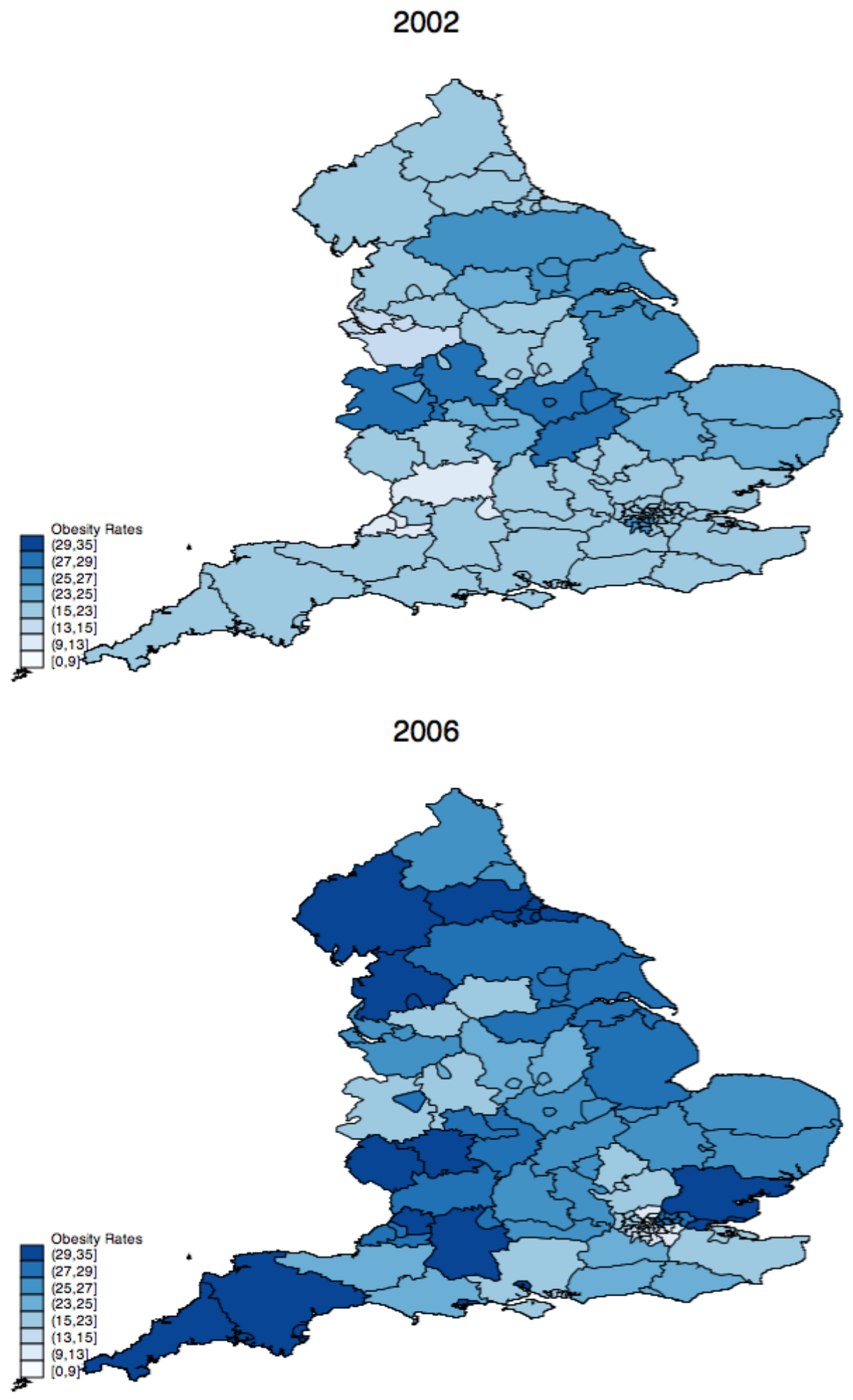
Table 1: Summary Statistics (2002-2006)

\begin{tabular}{lcccc}
\hline \hline & $(1)$ & $(2)$ & $(3)$ & $(4)$ \\
& All & Healthy & Overweight & Obese \\
mean & mean & mean \\
\hline male & 0.462 & 0.393 & 0.540 & 0.460 \\
age (last birthday) & 40.497 & 37.392 & 42.245 & 43.511 \\
self-assessed general health & 1.896 & 1.775 & 1.866 & 2.141 \\
marital & 0.859 & 0.867 & 0.857 & 0.846 \\
Education & 12.958 & 13.184 & 12.939 & 12.616 \\
rural & 0.129 & 0.129 & 0.133 & 0.125 \\
BMI & 26.864 & 22.499 & 27.273 & 34.112 \\
Drinking & 0.922 & 0.923 & 0.928 & 0.912 \\
N. of Cigarettes (daily) & 3.852 & 4.263 & 3.531 & 3.518 \\
Eating Habits & 3.448 & 3.395 & 3.544 & 3.431 \\
Dem. Group & 26.945 & 26.612 & 27.154 & 27.240 \\
Average BMI in HA & 26.824 & 26.802 & 26.828 & 26.857 \\
Perc. of Ob. in HA & 0.229 & 0.227 & 0.229 & 0.231 \\
Obese & 0.230 & & & \\
Overweight & 0.370 & & & \\
Healthy & 0.386 & & & \\
\hline Observations & 32935 & 12722 & 12195 & 7559 \\
\hline \hline
\end{tabular}


Table 2: Fairlie Decomposition 2002-2006

\begin{tabular}{|c|c|c|c|c|}
\hline & \multicolumn{4}{|c|}{ Obesity } \\
\hline & $\begin{array}{c}(1) \\
\text { Fairlie }\end{array}$ & $\begin{array}{c}(2) \\
\text { Fairlie }\end{array}$ & $\begin{array}{c}(3) \\
\text { Fairlie }\end{array}$ & $\begin{array}{c}(4) \\
\text { Fairlie }\end{array}$ \\
\hline Demographic & $\begin{array}{c}-0.0208^{* * *} \\
(0.0029)\end{array}$ & $\begin{array}{c}0.0006 \\
(0.0040)\end{array}$ & $\begin{array}{c}-0.0212^{* * *} \\
(0.0030)\end{array}$ & $\begin{array}{c}-0.0213^{* * *} \\
(0.0030)\end{array}$ \\
\hline SES & $\begin{array}{l}-0.0002 \\
(0.0009)\end{array}$ & $\begin{array}{c}0.0000 \\
(0.0003)\end{array}$ & $\begin{array}{c}-0.0004 \\
(0.0009)\end{array}$ & $\begin{array}{c}-0.0004 \\
(0.0009)\end{array}$ \\
\hline Education & $\begin{array}{c}0.0021^{* * *} \\
(0.0005)\end{array}$ & $\begin{array}{l}-0.0001 \\
(0.0004)\end{array}$ & $\begin{array}{c}0.0021^{* * *} \\
(0.0005)\end{array}$ & $\begin{array}{c}0.0021^{* * *} \\
(0.0005)\end{array}$ \\
\hline Health & $\begin{array}{l}-0.0004 \\
(0.0007)\end{array}$ & $\begin{array}{c}-0.0041^{* * *} \\
(0.0010)\end{array}$ & $\begin{array}{l}-0.0007 \\
(0.0007)\end{array}$ & $\begin{array}{l}-0.0008 \\
(0.0007)\end{array}$ \\
\hline Drinking & $\begin{array}{c}-0.0005^{*} \\
(0.0002)\end{array}$ & $\begin{array}{c}-0.0010^{*} \\
(0.0004)\end{array}$ & $\begin{array}{c}-0.0006^{*} \\
(0.0003)\end{array}$ & $\begin{array}{c}-0.0007^{*} \\
(0.0003)\end{array}$ \\
\hline N. of Cig. (daily) & $\begin{array}{l}-0.0003 \\
(0.0002)\end{array}$ & $\begin{array}{c}-0.0004^{*} \\
(0.0002)\end{array}$ & $\begin{array}{l}-0.0004 \\
(0.0002)\end{array}$ & $\begin{array}{l}-0.0004 \\
(0.0002)\end{array}$ \\
\hline Eating Habits & $\begin{array}{l}-0.0001 \\
(0.0013)\end{array}$ & $\begin{array}{l}-0.0001 \\
(0.0013)\end{array}$ & $\begin{array}{l}-0.0002 \\
(0.0014)\end{array}$ & $\begin{array}{l}-0.0003 \\
(0.0014)\end{array}$ \\
\hline Dem. Group & & $\begin{array}{c}-0.0163^{* * *} \\
(0.0026)\end{array}$ & & \\
\hline Average $\mathrm{BMI}$ in $\mathrm{HA}$ & & & $\begin{array}{c}-0.0082^{* *} \\
(0.0031)\end{array}$ & \\
\hline Perc. of Ob. in HA & & & & $\begin{array}{c}-0.0123^{* * *} \\
(0.0037)\end{array}$ \\
\hline Observations & 13521 & 13521 & 13521 & 13521 \\
\hline Food Prices & Yes & Yes & Yes & Yes \\
\hline Obesity (Early) & 0.2021 & 0.2021 & 0.2021 & 0.2021 \\
\hline Obesity (Late) & 0.2472 & 0.2472 & 0.2472 & 0.2472 \\
\hline Difference & -0.0451 & -0.0451 & -0.0451 & -0.0451 \\
\hline Tot Expl. Difference & -0.0202 & -0.0214 & -0.0295 & -0.0341 \\
\hline
\end{tabular}

Standard errors in parentheses

${ }^{*} p<0.05,{ }^{* *} p<0.01,{ }^{* * *} p<0.001$ 
Table 3: Fairlie Decomposition 2002-2006, by Gender

\begin{tabular}{|c|c|c|c|c|c|c|}
\hline & \multicolumn{6}{|c|}{ Obesity } \\
\hline & $(1)$ & $(2)$ & $(3)$ & $(4)$ & $(5)$ & $(6)$ \\
\hline & Men & Women & Men & Women & Men & Women \\
\hline Demographic & $\begin{array}{c}-0.0012 \\
(0.0069)\end{array}$ & $\begin{array}{c}0.0025 \\
(0.0050)\end{array}$ & $\begin{array}{c}-0.0294^{* * *} \\
(0.0049)\end{array}$ & $\begin{array}{c}-0.0136^{* * *} \\
(0.0038)\end{array}$ & $\begin{array}{c}-0.0299^{* * *} \\
(0.0049)\end{array}$ & $\begin{array}{c}-0.0137^{* * *} \\
(0.0038)\end{array}$ \\
\hline SES & $\begin{array}{c}0.0007 \\
(0.0008)\end{array}$ & $\begin{array}{l}-0.0002 \\
(0.0004)\end{array}$ & $\begin{array}{c}0.0008 \\
(0.0019)\end{array}$ & $\begin{array}{l}-0.0004 \\
(0.0008)\end{array}$ & $\begin{array}{c}0.0009 \\
(0.0019)\end{array}$ & $\begin{array}{c}-0.0005 \\
(0.0008)\end{array}$ \\
\hline Education & $\begin{array}{l}-0.0001 \\
(0.0006)\end{array}$ & $\begin{array}{c}0.0009 \\
(0.0005)\end{array}$ & $\begin{array}{c}0.0016 \\
(0.0008)\end{array}$ & $\begin{array}{l}0.0021^{* *} \\
(0.0007)\end{array}$ & $\begin{array}{c}0.0015 \\
(0.0008)\end{array}$ & $\begin{array}{l}0.0020^{* *} \\
(0.0007)\end{array}$ \\
\hline Health & $\begin{array}{c}-0.0042^{* *} \\
(0.0016)\end{array}$ & $\begin{array}{l}-0.0009 \\
(0.0015)\end{array}$ & $\begin{array}{c}-0.0024^{*} \\
(0.0012)\end{array}$ & $\begin{array}{l}0.0031^{* *} \\
(0.0011)\end{array}$ & $\begin{array}{c}-0.0024^{*} \\
(0.0012)\end{array}$ & $\begin{array}{l}0.0030^{* *} \\
(0.0011)\end{array}$ \\
\hline Drinking & $\begin{array}{l}-0.0012 \\
(0.0006)\end{array}$ & $\begin{array}{l}-0.0008 \\
(0.0005)\end{array}$ & $\begin{array}{l}-0.0007 \\
(0.0004)\end{array}$ & $\begin{array}{l}-0.0006 \\
(0.0004)\end{array}$ & $\begin{array}{l}-0.0009 \\
(0.0005)\end{array}$ & $\begin{array}{l}-0.0006 \\
(0.0004)\end{array}$ \\
\hline N. of Cig. (daily) & $\begin{array}{l}-0.0011 \\
(0.0006)\end{array}$ & $\begin{array}{l}-0.0004 \\
(0.0003)\end{array}$ & $\begin{array}{l}-0.0000 \\
(0.0004)\end{array}$ & $\begin{array}{l}-0.0008^{*} \\
(0.0004)\end{array}$ & $\begin{array}{l}-0.0002 \\
(0.0004)\end{array}$ & $\begin{array}{l}-0.0007^{*} \\
(0.0004)\end{array}$ \\
\hline Eating Habits & $\begin{array}{l}-0.0010 \\
(0.0015)\end{array}$ & $\begin{array}{c}0.0003 \\
(0.0012)\end{array}$ & $\begin{array}{c}-0.0018 \\
(0.0022)\end{array}$ & $\begin{array}{c}0.0005 \\
(0.0018)\end{array}$ & $\begin{array}{l}-0.0019 \\
(0.0022)\end{array}$ & $\begin{array}{c}0.0004 \\
(0.0019)\end{array}$ \\
\hline Dem. Group & $\begin{array}{c}-0.0217^{* * *} \\
(0.0050)\end{array}$ & $\begin{array}{c}-0.0118^{* * *} \\
(0.0029)\end{array}$ & & & & \\
\hline Average $\mathrm{BMI}$ in $\mathrm{HA}$ & & & $\begin{array}{c}-0.0152^{* * *} \\
(0.0041)\end{array}$ & $\begin{array}{l}-0.0016 \\
(0.0044)\end{array}$ & & \\
\hline Perc. of Ob. in HA & & & & & $\begin{array}{c}-0.0212^{* * *} \\
(0.0052)\end{array}$ & $\begin{array}{l}-0.0045 \\
(0.0052)\end{array}$ \\
\hline Observations & 6295 & 7226 & 6295 & 7226 & 6295 & 7226 \\
\hline Food Prices & Yes & Yes & Yes & Yes & Yes & Yes \\
\hline Obesity (Early) & 0.1939 & 0.2092 & 0.1939 & 0.2092 & 0.1939 & 0.2092 \\
\hline Obesity (Late) & 0.2540 & 0.2413 & 0.2540 & 0.2413 & 0.2540 & 0.2413 \\
\hline Difference & -0.0600 & -0.0321 & -0.0600 & -0.0321 & -0.0600 & -0.0321 \\
\hline Tot Expl. Difference & -0.0298 & -0.0104 & -0.0472 & -0.0115 & -0.0542 & -0.0146 \\
\hline
\end{tabular}

Standard errors in parentheses

${ }^{*} p<0.05,{ }^{* *} p<0.01,{ }^{* * *} p<0.001$ 
Table 4: Fairlie Decomposition 2002-2006, by Skill level (Male Only)

\begin{tabular}{lcccc}
\hline & \multicolumn{4}{c}{ Obesity } \\
\cline { 2 - 5 } & High & High & Medium-Low & Medium-Low \\
\hline Demographic & -0.0019 & $-0.0205^{* * *}$ & 0.0012 & $-0.0311^{* * *}$ \\
& $(0.0075)$ & $(0.0060)$ & $(0.0094)$ & $(0.0067)$ \\
Education & 0.0005 & 0.0006 & $-0.0024^{*}$ & -0.0004 \\
& $(0.0007)$ & $(0.0010)$ & $(0.0011)$ & $(0.0006)$ \\
Health & -0.0023 & 0.0011 & -0.0041 & -0.0031 \\
& $(0.0028)$ & $(0.0024)$ & $(0.0024)$ & $(0.0022)$ \\
Drinking & -0.0001 & -0.0002 & $-0.0020^{*}$ & -0.0011 \\
& $(0.0005)$ & $(0.0008)$ & $(0.0010)$ & $(0.0007)$ \\
N. of Cig. (daily) & -0.0011 & -0.0008 & -0.0010 & 0.0016 \\
Eating Habits & $(0.0010)$ & $(0.0009)$ & $(0.0011)$ & $(0.0008)$ \\
& -0.0005 & -0.0016 & -0.0008 & -0.0013 \\
Dem. Group & $(0.0025)$ & $(0.0036)$ & $(0.0017)$ & $(0.0022)$ \\
& $-0.0142^{* *}$ & & $-0.0245^{* * *}$ & \\
Average BMI in HA & $(0.0052)$ & & $(0.0067)$ & \\
& & $-0.0166^{*}$ & & $-0.0143^{* *}$ \\
Observations & & $(0.0076)$ & & $(0.0048)$ \\
Food Prices & 2419 & 2419 & 3876 & 3876 \\
Obesity (Early) & 0.1962 & 0.1962 & 0.1928 & 0.1928 \\
Obesity (Late) & 0.2394 & 0.2394 & 0.2644 & 0.2644 \\
Difference & -0.0432 & -0.0432 & -0.0716 & -0.0716 \\
Staspl. Difference & -0.0192 & -0.0383 & -0.0335 & -0.0497 \\
\hline
\end{tabular}

Standard errors in parentheses

${ }^{*} p<0.05,{ }^{* *} p<0.01,{ }^{* * *} p<0.001$ 
Table 5: Fairlie Decomposition 2002-2006, by Skill level (Male Only), Alternative social norm definition

\begin{tabular}{lcc}
\hline & \multicolumn{2}{c}{ Obesity } \\
\cline { 2 - 3 } & High & Medium-Low \\
\hline Demographic & -0.0166 & $-0.0320^{* * *}$ \\
& $(0.0117)$ & $(0.0068)$ \\
Education & 0.0005 & -0.0005 \\
& $(0.0009)$ & $(0.0006)$ \\
Health & 0.0008 & -0.0030 \\
& $(0.0024)$ & $(0.0023)$ \\
Drinking & -0.0002 & -0.0012 \\
& $(0.0008)$ & $(0.0007)$ \\
N. of Cig. (daily) & -0.0009 & 0.0013 \\
& $(0.0010)$ & $(0.0008)$ \\
Eating Habits & -0.0015 & -0.0013 \\
& $(0.0036)$ & $(0.0022)$ \\
Perc. of Ob. in HA & $-0.0210^{*}$ & $-0.0205^{* * *}$ \\
& $(0.0100)$ & $(0.0060)$ \\
\hline Observations & 2419 & 3876 \\
Food Prices & Yes & Yes \\
Obesity (Early) & 0.1962 & 0.1928 \\
Obesity (Late) & 0.2394 & 0.2644 \\
Difference & -0.0432 & -0.0716 \\
Tot Expl. Difference & -0.0388 & -0.0572 \\
\hline Standard & &
\end{tabular}

Standard errors in parentheses

${ }^{*} p<0.05,{ }^{* *} p<0.01,{ }^{* * *} p<0.001$ 
Table 6: Fairlie Decomposition 2002-2006, by Skill level (Female Only)

\begin{tabular}{|c|c|c|c|c|}
\hline & \multicolumn{4}{|c|}{ Obesity } \\
\hline & $\begin{array}{l}(1) \\
\text { High }\end{array}$ & $\begin{array}{c}(2) \\
\text { High }\end{array}$ & $\begin{array}{c}(3) \\
\text { Medium-Low }\end{array}$ & $\begin{array}{c}(4) \\
\text { Medium-Low }\end{array}$ \\
\hline Demographic & $\begin{array}{l}-0.0121 \\
(0.0122)\end{array}$ & $\begin{array}{c}-0.0175^{* *} \\
(0.0065)\end{array}$ & $\begin{array}{c}0.0060 \\
(0.0054)\end{array}$ & $\begin{array}{c}-0.0126^{* *} \\
(0.0048)\end{array}$ \\
\hline Education & $\begin{array}{c}0.0030 \\
(0.0017)\end{array}$ & $\begin{array}{l}0.0032^{* *} \\
(0.0012)\end{array}$ & $\begin{array}{l}-0.0005 \\
(0.0004)\end{array}$ & $\begin{array}{l}-0.0003 \\
(0.0004)\end{array}$ \\
\hline Health & $\begin{array}{c}0.0038 \\
(0.0025)\end{array}$ & $\begin{array}{l}0.0046^{*} \\
(0.0022)\end{array}$ & $\begin{array}{c}-0.0046^{*} \\
(0.0020)\end{array}$ & $\begin{array}{l}-0.0002 \\
(0.0015)\end{array}$ \\
\hline Drinking & $\begin{array}{c}0.0025 \\
(0.0023)\end{array}$ & $\begin{array}{c}0.0025 \\
(0.0025)\end{array}$ & $\begin{array}{l}-0.0009 \\
(0.0005)\end{array}$ & $\begin{array}{l}-0.0006 \\
(0.0005)\end{array}$ \\
\hline N. of Cig. (daily) & $\begin{array}{l}-0.0002 \\
(0.0026)\end{array}$ & $\begin{array}{l}-0.0002 \\
(0.0028)\end{array}$ & $\begin{array}{c}0.0009 \\
(0.0005)\end{array}$ & $\begin{array}{c}0.0006 \\
(0.0004)\end{array}$ \\
\hline Eating Habits & $\begin{array}{c}0.0024 \\
(0.0038)\end{array}$ & $\begin{array}{c}0.0025 \\
(0.0042)\end{array}$ & $\begin{array}{l}-0.0002 \\
(0.0011)\end{array}$ & $\begin{array}{l}-0.0002 \\
(0.0018)\end{array}$ \\
\hline Dem. Group & $\begin{array}{l}-0.0031 \\
(0.0070)\end{array}$ & & $\begin{array}{c}-0.0143^{* * *} \\
(0.0033)\end{array}$ & \\
\hline Average BMI in HA & & $\begin{array}{c}0.0099 \\
(0.0102)\end{array}$ & & $\begin{array}{l}-0.0048 \\
(0.0048)\end{array}$ \\
\hline Observations & 2382 & 2382 & 4844 & 4844 \\
\hline Food Prices & Yes & Yes & Yes & Yes \\
\hline Obesity (Early) & 0.1938 & 0.1938 & 0.2158 & 0.2158 \\
\hline Obesity (Late) & 0.2132 & 0.2132 & 0.2564 & 0.2564 \\
\hline Difference & -0.0194 & -0.0194 & -0.0407 & -0.0407 \\
\hline Tot Expl. Difference & -0.0041 & 0.0048 & -0.0135 & -0.0181 \\
\hline
\end{tabular}

Standard errors in parentheses

${ }^{*} p<0.05,{ }^{* *} p<0.01,{ }^{* * *} p<0.001$ 
Table 7: Fairlie Decomposition 2002-2006, by Skill level (Female Only), Alternative social norm definition

\begin{tabular}{lcc}
\hline & \multicolumn{2}{c}{ Obesity } \\
\cline { 2 - 3 } & High & Medium-Low \\
\hline Demographic & $-0.0180^{* *}$ & $-0.0213^{* * *}$ \\
SES & $(0.0066)$ & $(0.0058)$ \\
& 0.0033 & 0.0024 \\
Education & $(0.0086)$ & $(0.0054)$ \\
& $0.0030^{*}$ & -0.0002 \\
Health & $(0.0012)$ & $(0.0003)$ \\
& $0.0045^{*}$ & -0.0003 \\
Drinking & $(0.0022)$ & $(0.0015)$ \\
N. of Cig. (daily) & 0.0025 & -0.0006 \\
& $(0.0024)$ & $(0.0005)$ \\
Eating Habits & -0.0001 & 0.0004 \\
& $(0.0028)$ & $(0.0004)$ \\
Perc. of Ob. in HA & 0.0025 & -0.0002 \\
& $(0.0041)$ & $(0.0018)$ \\
Observations & 0.0074 & -0.0089 \\
Food Prices & $(0.0116)$ & $(0.0059)$ \\
Obesity (Early) & 2382 & 4844 \\
Obesity (Late) & Yes & Yes \\
Difference & 0.1938 & 0.2158 \\
Tot Expl. Difference & 0.2132 & 0.2564 \\
Stard & -0.0194 & -0.0407 \\
& 0.0053 & -0.0287 \\
\hline
\end{tabular}

Standard errors in parentheses

${ }^{*} p<0.05,{ }^{* *} p<0.01,{ }^{* * *} p<0.001$ 